\title{
Ion-Irradiated Damage in Semiconductors Visualized by Means of Low-kV Scanning Electron Microscopy
}

\author{
Iwona Jozwik $^{1,2}$, Adam Barcz ${ }^{3}$, Ewa Dumiszewska ${ }^{1}$ and Elzbieta Dabrowska ${ }^{1}$ \\ 1. Institute of Electronic Materials Technology, Warsaw, Poland \\ 2. National Centre for Nuclear Research, NOMATEN Centre of Excellence, Swierk-Otwock, Poland \\ 3. Institute of Electron Technology/Institute of Physics PAS, Warsaw, Poland
}

Low-voltage scanning electron microscopy is a technique used in semiconductor technology to detect contrast originating from the active dopant distribution and concentration when imaging of cleaved structures of electronic devices [1,2]. In this paper we show, that it may be used as well to visualize highly insulating channels produced by ion damaging of semiconductors [3]. The technique of implant isolation was found applicable to the separation of discrete electronic devices such as HEMT's, lasers or power MESFET's manufactured on a semiconductor wafer. The unrivalled advantage of the low-kV SEM imaging technique of such structures lies in its immediate generation of useful two dimensional damage visualization without complex sample preparation. Since the mechanism of passive voltage contrast in SEM images related to local differences in the surface potential is well known [4,5], the origin and interpretation of contrast based on local changes in the electrical properties resulting from the ion-irradiation damage occurring in the material are still lacking. The main aim of the paper is to present the mechanism Damage-Induced Voltage Alteration (DIVA) contrast in SEM images, which is based on the charging effect resulting from ion-irradiation damage and subsequent formation of highly resistive regions within the semiconductor.

The $\mathrm{Al}_{0.55} \mathrm{Ga}_{0.45} \mathrm{As}$ (p-type and n-type) layers of approximate thickness of $3 \mu \mathrm{m}$ grown over the GaAs substrates by means of MOCVD (metal-organic chemical vapour deposition) technique were subjected to ion irradiation through metallic masks with $\mathrm{He}^{+}$ions of various fluencies ranging from 8e12 to 8e13 $\mathrm{cm}^{-2}$ after acceleration by $760 \mathrm{kV}$. The final specimens were prepared by simple cleavage and examined in the scanning electron microscope at low energy operation (Auriga CrossBeam Workstation (Carl Zeiss)).

The point defects and defect agglomerates affect the electrical properties of the semiconductor. The level of damage translates into the increase of local resistivity of the material resulting from the conductivity compensation [6,7]. Electronic contrast developing on the irradiated areas provides information on the local resistivity - a quantity decisive for the ultimate effectiveness of device isolation. In the case studied, the $\mathrm{He}^{+}$-irradiated areas appear dark or almost black in the SE1 image (Fig.1). The resultant damage-induced contrast is bound to the yield of SE and presents a fluencedependent character (Fig. 2). The DIVA contrast is considered to be driven by redistribution of the SEs affected by the local fields on the specimen surface and alteration of the original surface potential (see explanation in Fig. 3). The applicability of the technique may safely be generalized to other semiconductors where electrical isolation of devices fabricated in planar technology is needed [8].

\section{References:}

[1] M M Gomati and T C R Wells, Appl. Phys. Lett. 79 (2001), p. 2931.

[2] P G Merli et al, Appl. Phys. Lett. 86 (2005), p. 101916. 
[3] I Jóźwik et al, ECS J. Solid State Sci. Technol. 6(7) (2017), p. 415.

[4] O D Patterson et al, Proceedings of The 17th Annual SEMI/IEEE ASMC 2006 Conference, doi:10.1109/ASMC.2006.1638778.

[5] R Rosenkranz, J Mater Sci: Mater Electron 22 (2011), p.1523.

[6] V N Brudnyi and M A Krivov, Soviet Physics Journal 23 (1980), p. 45.

[7] S J Pearton, Mater. Sci. Rep. 4 (1990), p. 313.

[8] The authors acknowledge funding from the National Science Centre, Poland, Grant Number 2017/27/B/ST8/01158.

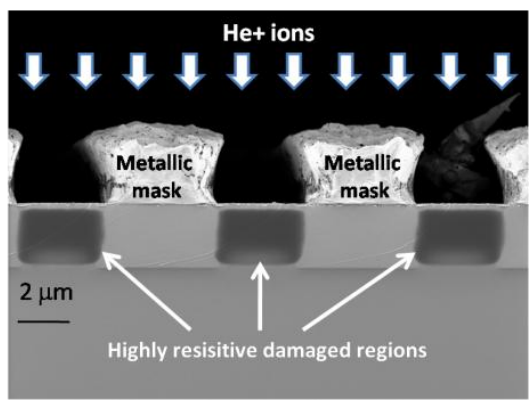

Figure 1. SEM image of AlGaAs layer cross-section with visible ion-damaged regions.

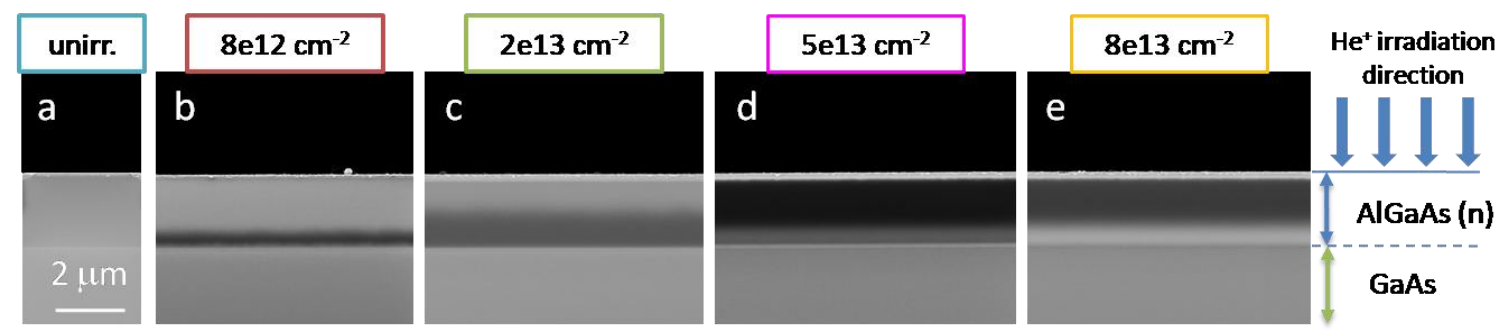

Figure 2. Low-kV SEM images of the cleaved cross-sections of He ${ }^{+}$-irradiated n-type AlGaAs/GaAs samples; the damage-related contrast evolution with increasing fluencies is evident.

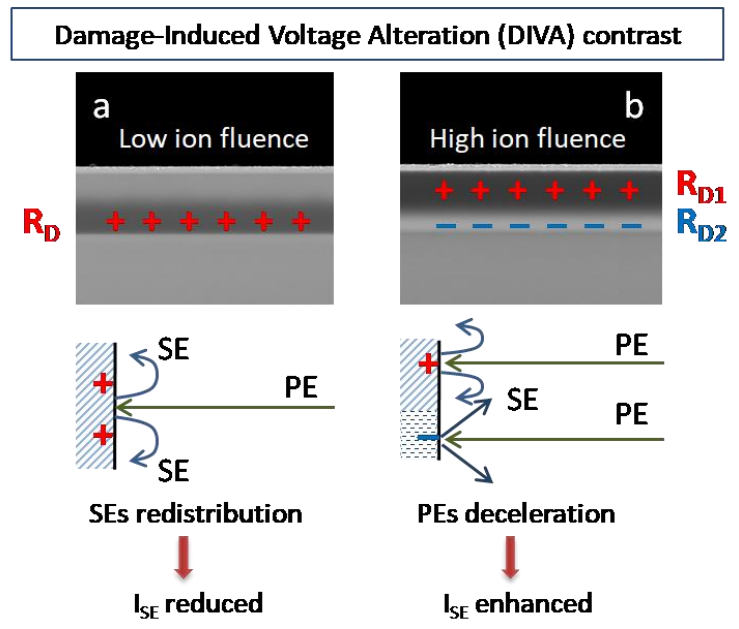

Figure 3. The DIVA contrast mechanism explained: (a) lower fluence: damaged region of resistivity $R_{D}$ is charging positively, thus diminishing SE emission - the region appears black in SEM image; (b) higher fluence: resistivity driven by higher level of damage collects negative charge, consequently repelling PE and increasing SE emission - brightening in the SEM image. 